\title{
The Very First Stars: Formation and Reionization of the Universe
}

\author{
Volker Bromm ${ }^{1}$ \\ ${ }^{1}$ Department of Astronomy, University of Texas, Austin, TX 78712, U.S.A.
}

\begin{abstract}
One of the key challenges for the next 10 years is to understand the first sources of light, the first stars and possibly accreting black holes. Their formation ended the cosmic dark ages at redshifts $z \simeq 20-30$, and signaled the transition from the simple initial state of the universe to one of ever increasing complexity. We here review recent progress in understanding the formation process of the first stars with numerical simulations, starting with cosmological initial conditions and modelling the detailed physics of accretion. Once formed, the first stars exerted crucial feedback on the primordial intergalactic medium, due to their input of radiation and of heavy chemical elements in the wake of supernova explosions. The current theoretical model posits that the first stars were predominantly very massive, typically $\sim 100 M_{\odot}$. Our predictions will be tested with upcoming near-infrared observatories, such as the James Webb Space Telecope, in the decade ahead.
\end{abstract}

Keywords. cosmology: theory, early universe - galaxies: formation — stars: formation, supernovae

\section{Introduction}

An important open question in cosmology is to understand how and when the cosmic dark ages ended (Bromm et al. 2009). Within the current $\Lambda$ CDM paradigm, the first stars, the so-called Population III (Pop III), are predicted to have formed at redshifts $z \simeq 20-30$. Their emergence signals the rapid transformation of the universe into an increasingly complex, hierarchical system, due to the energy and heavy element input from Pop III stars and accreting black holes (Barkana \& Loeb 2001; Bromm \& Larson 2004; Ciardi \& Ferrara 2005; Miralda-Escudé 2003). Currently, we can directly probe the state of the universe roughly a million years after the Big Bang by detecting the temperature anisotropies in the cosmic microwave background (CMB), thus providing us with the initial conditions for subsequent structure formation. Complementary to the CMB observations, we can probe cosmic history all the way from the present-day universe to roughly a billion years after the Big Bang, using the best available ground- and spacebased telescopes. In between lies the remaining frontier, and the first stars and galaxies are the sign-posts of this early, formative epoch.

To simulate the build-up of the first stellar systems, we have to address the feedback from the very first stars on the surrounding intergalactic medium (IGM), and the formation of the second generation of stars out of material that was influenced by this feedback. There are a number of reasons why addressing the feedback from the first stars and understanding second-generation star formation is crucial:

(i) The first steps in the hierarchical build-up of structure provide us with a simplified laboratory for studying galaxy formation, which is one of the main outstanding problems in cosmology.

(ii) The initial burst of Pop III star formation may have been rather brief due to the strong negative feedback effects that likely acted to self-limit this formation mode 
(Greif \& Bromm 2006; Yoshida et al. 2004). Second-generation star formation, therefore, might well have been cosmologically dominant compared to Pop III stars.

(iii) A subset of second-generation stars, those with masses below $\simeq 1 M_{\odot}$, would have survived to the present day. Surveys of extremely metal-poor Galactic halo stars therefore provide an indirect window into the Pop III era by scrutinizing their chemical abundance patterns, which reflect the enrichment from a single, or at most a small multiple of, Pop III SNe (Beers \& Christlieb 2005; Frebel et al. 2007; Karlsson et al. 2008). Stellar archaeology thus provides unique empirical constraints for numerical simulations, from which one can derive theoretical abundance patterns to be compared with the data.

Existing and planned observatories, such as HST, Keck, VLT, and the James Webb Space Telescope (JWST), planned for launch around 2014, yield data on stars and quasars less than a billion years after the Big Bang. The ongoing Swift gamma-ray burst (GRB) mission provides us with a possible window into massive star formation at the highest redshifts (Lamb \& Reichart 2000; Bromm \& Loeb 2002, 2006). Measurements of the nearIR cosmic background radiation, both in terms of the spectral energy distribution and the angular fluctuations provide additional constraints on the overall energy production due to the first stars (Santos et al. 2002; Magliocchetti et al. 2003; Dwek et al. 2005; Fernandez \& Komatsu 2006; Kashlinsky et al. 2005). Understanding the formation of the first sources of light is thus of great interest to observational studies conducted both at high redshifts and in our local Galactic neighborhood.

\section{Primordial Star Formation}

The first stars in the universe formed a few 100 Myr after the Big Bang, when the primordial gas was first able to cool and collapse into dark matter (DM) minihalos (see Fig. 1) with masses of the order of $10^{6} M_{\odot}$ (Abel et al. 2002; Bromm et al. 2002; Yoshida et al. 2006, 2008). These stars are believed to have been very massive, with masses of the order of $100 M_{\odot}$, owing to the limited cooling ability of primordial gas in minihalos via the radiation from $\mathrm{H}_{2}$ molecules. While the initial conditions for the formation of the very first stars are known from precision measurements of cosmological parameters (Spergel et al. 2007), the situation for the subsequent generations of stars is much more compex. It has become evident that Pop III star formation might actually consist of two distinct modes: one where the primordial gas collapses into a DM minihalo, and one where the metal-free gas becomes significantly ionized prior to the onset of gravitational runaway collapse (Johnson \& Bromm 2006). To clearly indicate that both modes pertain to metal-free star formation, we here follow the new classification scheme suggested by Chris McKee (see McKee \& Tan 2008; Johnson et al. 2008). Within this scheme, the minihalo Pop III mode is termed Pop III.1, whereas the second mode is called Pop III.2.

Numerical simulations have converged on characterising the properties of the Jeansunstable cloud, the immediate progenitor for Pop III star formation, and the initial runaway collapse towards the formation of an optically-thick protostellar core. The latter has an initial mass, $\sim 10^{-2} M_{\odot}$, which is very similar to the present-day case (Yoshida et al. 2008). The build-up process depends on the detailed physics of accretion and protostellar feedback. Based on idealized models, it has been argued that the growth of a Pop III star proceeds in a disk-like fashion, again similar to the Pop I situation (Tan \& McKee 2004; McKee \& Tan 2008). Only recently has it become feasible to study the accretion process within the context of realistic cosmological initial conditions (Stacy et al. 2009). The emerging picture is that of a disk growing around a central dominant protostar until it becomes gravitationally unstable and fragments (see Fig. 2). Other simulations as well have found signs of binary, or small multiple system, formation 

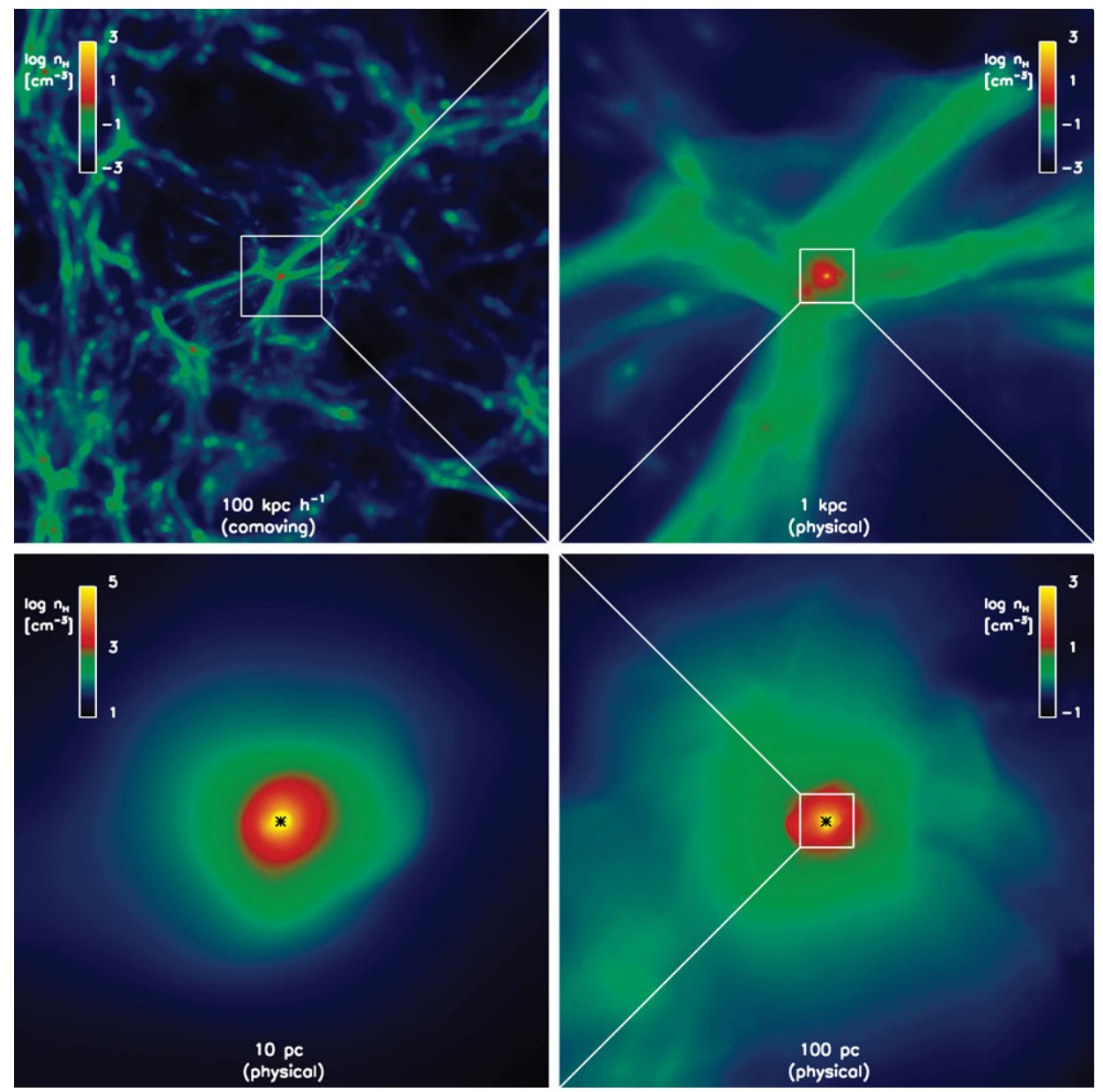

Figure 1. Cosmological environment for Pop III star formation (from Stacy et al. 2009). Shown is the gas density distribution on progressively smaller scales, as labeled in each panel. In the bottom two panels, the asterisk denotes the location of the first sink formed, which acts as a proxy for the growing protostar. The top two panels show the emergence of a minihalo, inside of which the dissipating gas assumes a quasi-hydrostatic morphology, just prior to the onset of runaway collapse.

(Turk et al. 2009). Again, the Pop III case might be less different from present-day massive star formation as previously thought. However, the task still remains to couple the 3-dimensional Pop III assembly with the radiation-hydrodynamics of protostellar feedback. It is to be expected that such radiative effects will hinder fragmentation into secondary clumps, once the first few protostars have emerged.

While the formation of the very first, Pop III.1, stars in minihalos relied on $\mathrm{H}_{2}$ cooling, the HD molecule can play an important role in the cooling of primordial gas in several situations, allowing the temperature to drop well below $200 \mathrm{~K}$ (Abel et al. 2002; Bromm et al. 2002). In turn, this efficient cooling may lead to the formation of primordial stars with masses of the order of $10 M_{\odot}$, the so-called Pop III.2 (Johnson \& Bromm 2006). In general, the formation of $\mathrm{HD}$, and the concomitant cooling that it provides, is found to occur efficiently in primordial gas which is strongly ionized, owing ultimately to the high abundance of electrons which serve as catalyst for molecule formation in the early 

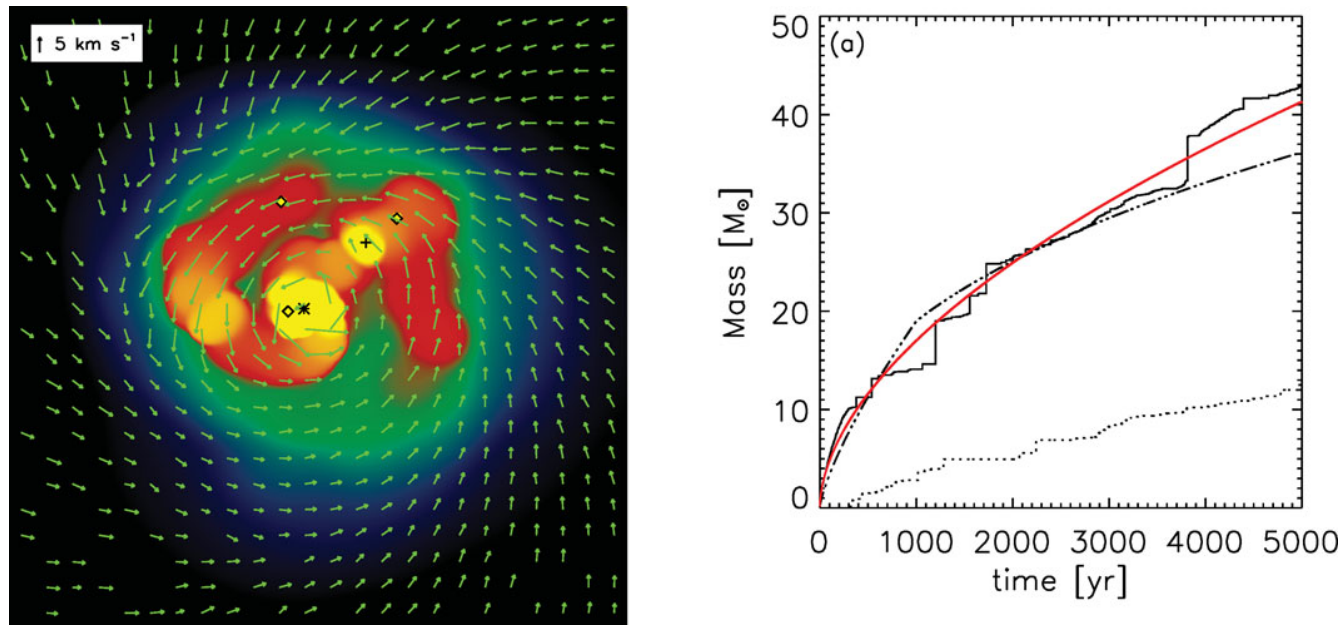

Figure 2. Disk formation and protostellar accretion (from Stacy et al. 2009). Left panel: Velocity field of the gas in the center of a minihalo. Sinks are denoted as follows: The asterisk marks the location of the most massive sink, crosses that of the second most massive one, and diamonds those of the other, smaller sinks. Shown is the situation $5000 \mathrm{yr}$ after initial sink formation. At this point, an ordered, nearly Keplerian velocity structure has been established within the disk. Right panel: Sink mass vs. time. The solid line shows the mass of the first sink particle, fitted by a power law according to $M \propto t^{0.55}$ (red line). The dash-dotted line depicts the accretion history found in Bromm \& Loeb (2004). The dotted line traces the mass growth of the second largest sink. It is evident that the sinks grow to masses $\gtrsim 10 M_{\odot}$ within a few $1,000 \mathrm{yr}$.

universe (Shapiro \& Kang 1987). Efficient cooling by HD can be triggered within the relic H II regions that surround Pop III.1 stars at the end of their brief lifetimes (Alvarez et al. 2006), owing to the high electron fraction that persists in the gas as it cools and recombines (Johnson et al. 2007; Yoshida et al. 2007b). The efficient formation of HD can also take place when the primordial gas is collisionally ionized, such as behind the shocks driven by the first SNe or in the virialization of massive DM halos (Machida et al. 2005; Johnson \& Bromm 2006; Shchekinov \& Vasiliev 2006).

There might thus be a progression of characteristic masses of the various stellar populations that form in the early universe. In the wake of Pop III.1 stars (typically with $M_{*} \sim 100 M_{\odot}$ ) formed in DM minihalos, Pop III.2 star formation (with $M_{*} \sim 10 M_{\odot}$ ) ensues in regions which have been previously ionized, typically associated with relic H II regions left over from massive Pop III.1 stars collapsing to black holes, while even later, when the primordial gas is locally enriched with metals, Pop II (with $M_{*} \sim 1 M_{\odot}$ ) stars begin to form (Bromm \& Loeb 2003; Greif \& Bromm 2006). Recent simulations confirm this picture, as Pop III.2 star formation ensues in relic H II regions in well under a Hubble time, while the formation of Pop II stars after the first SN explosions is delayed by more than a Hubble time (Greif et al. 2007; Yoshida et al. 2007a,b; but see Whalen et al. 2008).

\section{Towards the First Galaxies}

How massive were the first galaxies, and when did they emerge? Theory predicts that DM halos containing a mass of $\sim 10^{8} M_{\odot}$ and collapsing at $z \sim 10$ were the hosts for the first bona fide galaxies. These dwarf systems are special in that their associated virial temperature exceeds the threshold, $\sim 10^{4} \mathrm{~K}$, for cooling due to atomic hydrogen ( $\mathrm{Oh}$ \& Haiman 2002). These so-called 'atomic-cooling halos' did not rely on the presence of 
molecular hydrogen to enable cooling of the primordial gas. In addition, their potential wells were sufficiently deep to retain photoheated gas, in contrast to the shallow potential wells of minihalos (Dijkstra et al. 2004). These are arguably minimum requirements to set up a self-regulated process of star formation that comprises more than one generation of stars, and is embedded in a multi-phase interstellar medium.

One of the important consequences of atomic cooling is the softening of the equation of state below the virial radius, allowing a fraction of the potential energy to be converted into kinetic energy (Wise \& Abel 2007; Greif et al. 2008). This implies that perturbations in the gravitational potential can generate turbulent motions on galactic scales, which are then transported to the centre of the galaxy. In this context the distinction between two fundamentally different modes of accretion becomes important (Greif et al. 2008). Gas accreted directly from the IGM is heated to the virial temperature and comprises the sole channel of inflow until cooling in filaments becomes important. This mode is termed hot accretion, and dominates in low-mass haloes at high redshift. The formation of the virial shock and the concomitant heating in an atomic cooling halo are visible in Fig. 3. The second mode, termed cold accretion, becomes important as soon as filaments are massive enough to enable molecule reformation, which allows the gas to cool and flow into the central regions of the nascent galaxy with high velocities. Although the assembly of the first galaxies provides us with an idealized laboratory for galaxy formation in general, the degree of complexity that is exhibited in the corresponding merger tree is already considerable. Still, current supercomputer simulations have just reached the capacity to address the first galaxy formation process in an a-priori fashion, one star at a time.

Cold accretion is a viable agent for driving turbulence, due to the large amount of kinetic energy it brings to the center of the galaxy. Two physically distinct mechanisms are responsible for creating shocks (Greif et al. 2008). The virial shock forms where the ratio of infall velocity to local sound speed approaches unity, while a multitude of unorganized shocks forms near the center of the galaxy and is mostly caused by accretion of cold, high-velocity gas from filaments. These are more pronounced than the virial shock and have a significantly higher angular component. They create transitory density perturbations that could in principle become Jeans-unstable and trigger the gravitational collapse of individual clumps. In concert with metal enrichment by previous star formation in minihaloes, metal mixing in the first galaxies will likely be highly efficient and could lead to the formation of the first low-mass star clusters (Clark et al. 2008), in extreme cases possibly even to metal-poor globular clusters (Bromm \& Clarke 2002). Some of the extremely iron-deficient, but carbon and oxygen-enhanced stars observed in the halo of the Milky Way may thus have formed as early as redshift $z \simeq 10$ (Karlsson et al. 2008).

\section{Outlook}

Understanding the formation of the first sources of light marks the frontier of highredshift structure formation. It is crucial to predict their properties in order to develop the optimal search and survey strategies for the JWST. Whereas ab-initio simulations of the very first stars can be carried out from first principles, and with virtually no free parameters, one faces a much more daunting challenge with the first galaxies. Now, the previous history of star formation has to be considered, leading to enhanced complexity in the assembly of the first galaxies. One by one, all the complex astrophysical processes that play a role in more recent galaxy formation appear back on the scene. Among them are external radiation fields, comprising UV and X-ray photons, and possibly cosmic 

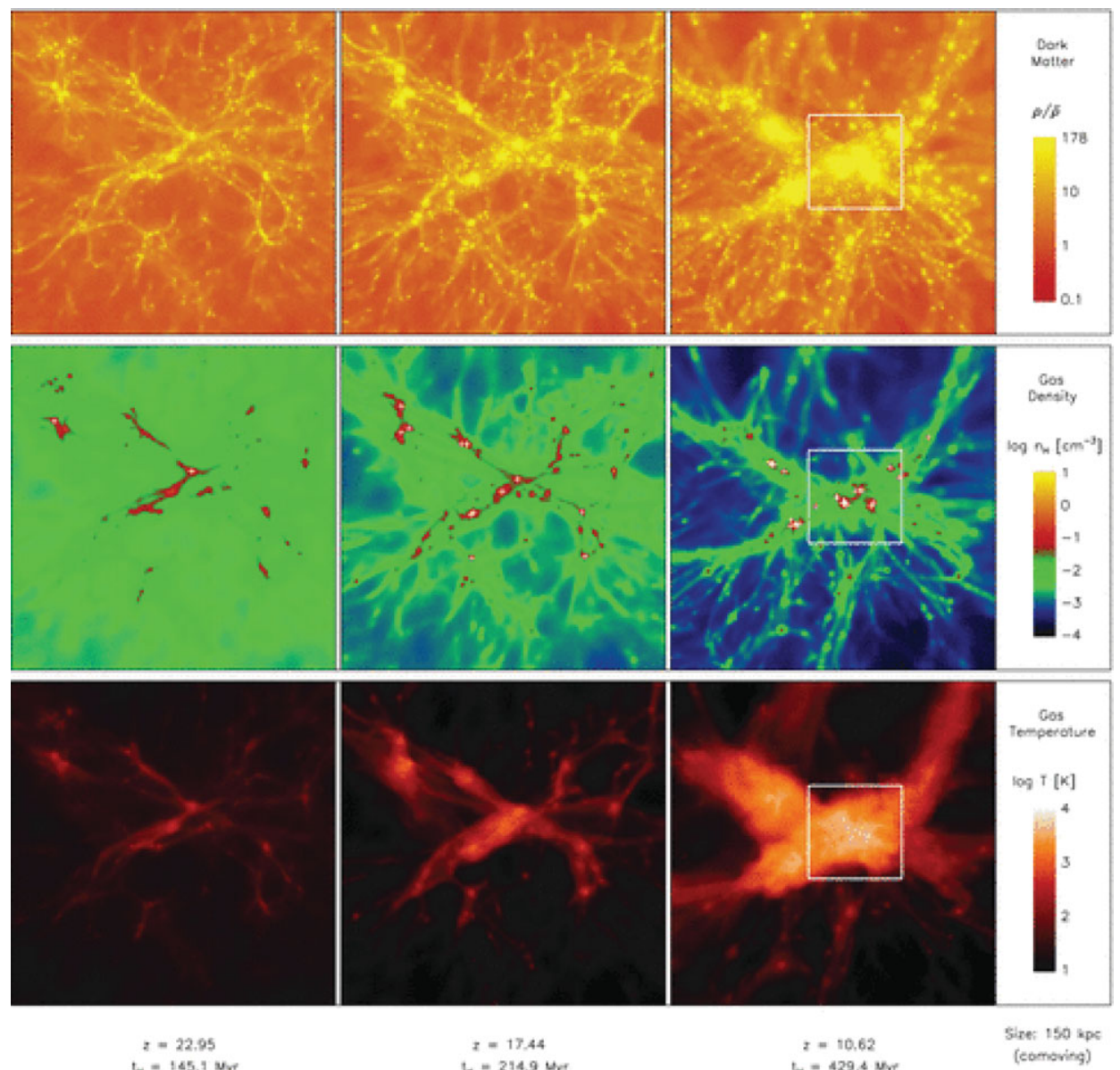

$z=17,44$

$4=214.9 u_{\text {ref }}$

$z=10,62$

Sire: $150 \mathrm{kps}$ (eomoving)

Figure 3. Assembly of the first galaxy (from Greif et al. 2008). Gravity is assembling the dark matter (top row) and pristine gas (middle row) into a primordial galaxy. Shown is the evolution at three different stages (from left to right). White crosses indicate individual Pop III stars, which form prior to the first galaxy. The assembly process is accompanied by powerful virialization shocks that heat up the infalling gas to $>10^{4} \mathrm{~K}$ (bottom row).

rays produced in the wake of the first SNe. There will be metal-enriched pockets of gas which could be pervaded by dynamically non-negligible magnetic fields, together with turbulent velocity fields built up during the virialization process. However, the goal of making useful predictions for the first stars and galaxies is now clearly drawing within reach, and the pace of progress is likely to be rapid. A few years from now we should have a better idea of whether our theoretical ideas are on track, or need to be refined.

\section{Acknowledgements}

I would like to thank the organizers for putting together a stimulating program of talks and discussions, which gave me numerous ideas for future exploration. Support from NSF grant AST-0708795 and NASA ATFP grant NNX08AL43G is gratefully acknowledged. The simulations presented here were carried out at the Texas Advanced Computing Center (TACC). 


\section{References}

Abel, T., Bryan, G. L., \& Norman, M. L. 2002, Science, 295, 93

Alvarez, M. A., Bromm, V., \& Shapiro, P. R. 2006, ApJ, 639, 621

Barkana, R. \& Loeb, A. 2001, Phys. Rep., 349, 125

Beers, T. C. \& Christlieb, N. 2005, ARA\&A, 43, 531

Bromm, V. \& Clarke, C. J. 2002, ApJ, 566, L1

Bromm, V., Coppi, P. S., \& Larson, R. B. 2002, ApJ, 564, 23

Bromm, V. \& Larson, R. B. 2004, ARA\&A, 42, 79

Bromm, V. \& Loeb, A. 2002, ApJ, 575, 111

-. 2003, Nature, 425, 812

-. 2004, New Astronomy, 9, 353

-. 2006, ApJ, 642, 382

Bromm, V., Yoshida, N., Hernquist, L., \& McKee, C. F. 2009, Nature, 459, 49

Ciardi, B. \& Ferrara, A. 2005, Space Science Reviews, 116, 625

Clark, P. C., Glover, S. C. O., \& Klessen, R. S. 2008, ApJ, 672, 757

Dijkstra, M., Haiman, Z., Rees, M. J., \& Weinberg, D. H. 2004, ApJ, 601, 666

Dwek, E., Arendt, R. G., \& Krennrich, F. 2005, ApJ, 635, 784

Fernandez, E. R. \& Komatsu, E. 2006, ApJ, 646, 703

Frebel, A., Johnson, J. L., \& Bromm, V. 2007, MNRAS, 380, L40

Greif, T. H. \& Bromm, V. 2006, MNRAS, 373, 128

Greif, T. H., Johnson, J. L., Bromm, V., \& Klessen, R. S. 2007, ApJ, 670, 1

Greif, T. H., Johnson, J. L., Klessen, R. S., \& Bromm, V., 2008, MNRAS, 387, 1021

Johnson, J. L. \& Bromm, V. 2006, MNRAS, 366, 247

Johnson, J. L., Greif, T. H., \& Bromm, V. 2007, ApJ, 665, 85

Johnson, J. L., Greif, T. H., \& Bromm, V. 2008, MNRAS, 388, 26

Karlsson, T., Johnson, J. L., \& Bromm, V. 2008, ApJ, 679, 6

Kashlinsky, A., Arendt, R. G., Mather, J., \& Moseley, S. H. 2005, Nature, 438, 45

Lamb, D. Q. \& Reichart, D. E. 2000, ApJ, 536, 1

Machida, M. N., Tomisaka, K., Nakamura, F., \& Fujimoto, M. Y. 2005, ApJ, 622, 39

Magliocchetti, M., Salvaterra, R., \& Ferrara, A. 2003, MNRAS, 342, L25

McKee C. F. \& Tan, J. C. 2008, ApJ, 681, 771

Miralda-Escudé, J. 2003, Science, 300, 1904

Oh, S. P. \& Haiman, Z. 2002, ApJ, 569, 558

Santos, M. R., Bromm, V., \& Kamionkowski, M. 2002, MNRAS, 336, 1082

Shapiro, P. R. \& Kang, H. 1987, ApJ, 318, 32

Shchekinov, Y. A. \& Vasiliev, E. O. 2006, MNRAS, 368, 454

Spergel, D. N. et al. 2007, ApJS, 170, 377

Stacy, A. R., Greif, T. H., \& Bromm, V. 2009, MNRAS, submitted (arXiv:0908.0712)

Tan, J. C. \& McKee C. F. 2004, ApJ, 603, 383

Turk, M. J., Abel, T., \& O'Shea, B. 2009, Science, 325, 601

Whalen, D., van Veelen, B., O'Shea, B. W., \& Norman, M. L. 2008, ApJ, 682, 49

Wise, J. H. \& Abel, T. 2007, ApJ, 665, 899

Yoshida, N., Bromm, V., \& Hernquist, L. 2004, ApJ, 605, 579

Yoshida, N., Oh, S. P., Kitayama, T., \& Hernquist, L. 2007a, ApJ, 663, 687

Yoshida, N., Omukai, K., \& Hernquist, L. 2007b, ApJ, 667, L117

Yoshida, N., Omukai, K., Hernquist, L., \& Abel, T. 2006, ApJ, 652, 6

Yoshida, N., Omukai, K., \& Hernquist, L. 2008, Science, 321, 669 